\title{
Argumentos para a Inviabilidade Prática de uma Estratégia de Escalonamento para Redes Sem Fio sob o Modelo SINR
}

\author{
Fábio Engel de Camargo ${ }^{1,2}$, Elias P. Duarte Jr. ${ }^{2}$ \\ ${ }^{1}$ Universidade Tecnológica Federal do Paraná (UTFPR) \\ R. Cristo Rei, 19 Toledo 85902-490 PR \\ ${ }^{2}$ Departamento de Informática - Universidade Federal do Paraná (UFPR) \\ Caixa Postal 19018 Curitiba 81531-990 PR \\ fabioedutfpr.edu.br, elias@inf.ufpr.br
}

\begin{abstract}
By considering the effects of cumulative interference on signal reception by the various devices connected to a wireless network, the SINR model (Signal-to-Interference-plus-Noise Ratio) has gained prominence. The model allows spatial reuse, i.e. multiple devices within the same range can communicate concurrently. The scheduling strategy by Halldorsson et al. [Halldorsson et al. 2017] allows the definition of which devices can communicate at subsequent time intervals. The strategy assumes a dense 1-hop wireless network, i.e.: all devices are within their respective ranges. Simulation experiments have indicated that there are obstacles that make it difficult to use the scheduling strategy in practice. We present two hypotheses that we believe reflect the reasons that make it difficult to be applied in real networks.
\end{abstract}

Resumo. Por considerar os efeitos da interferência cumulativa na recepção do sinal pelos diversos dispositivos conectados a uma rede sem fio, o modelo SINR (Signal-to-Interference-plus-Noise Ratio) tem ganhado destaque. O modelo permite o reuso espacial, que possibilita a comunicação concorrente de múltiplos dispositivos na mesma área de alcance. A estratégia de escalonamento de Halldórsson e outros [Halldórsson et al. 2017] permite definir quais dispositivos podem comunicar em quais intervalos de tempo. A proposta assume uma rede sem fio de 1-hop densa, isto é: todos os dispositivos estão dentro das respectivas áreas de alcance. A realização de experimentos de simulação indicou que há empecilhos que dificultam a utilização da estratégia na prática. Apresentamos duas hipóteses que acreditamos refletem os motivos que dificultam sua utilização em redes reais.

\section{Introdução}

O modelo SINR (Signal-to-Interference-plus-Noise Ratio) se destaca por consolidar uma boa representação do comportamento das redes de comunicação sem fio [Halldórsson and Tonoyan 2019]. O modelo considera os efeitos da interferência cumulativa na recepção do sinal pelos diversos dispositivos conectados à rede. A utilização deste modelo permite que sejam desenvolvidas estratégias que façam proveito do reuso espacial, que possibilita, sob determinadas condições, a comunicação concorrente de múltiplos dispositivos que se encontram na mesma área de alcance. 
A comunicação no modelo SINR deve ser precedida por uma etapa de escalonamento, em que se determina quais dispositivos podem comunicar em quais intervalos de tempo. A estratégia de escalonamento de Halldórsson e outros [Halldórsson et al. 2017] provê um algoritmo de escalonamento para redes sem fio densas que baseia-se no mecanismo de acesso TDMA (Time Division Multiple Access) para escalonar as transmissões. A proposta assume uma rede densa de 1-hop: todos os dispositivos estão mutuamente sob alcance de transmissão.

O algoritmo de escalonamento organiza os dispositivos em uma chamada "árvore de torneio" (descrita adiante no artigo). A árvore é construída a partir de um algoritmo que explora a relação SINR em transmissões simultâneas para criar um grafo que conecta dispositivos que se encontram a curtas distâncias geográficas entre si. Sobre este grafo, aplica-se um algoritmo de coloração como método de escalonamento de transmissões.

Para o desenvolvimento do presente trabalho foi implementado um simulador de redes sem fio sob o modelo SINR. A implementação fez uso do simulador OMNET $++^{1}$, em conjunto ao framework INET $^{2}$. No entanto, a simulação da estratégia de escalonamento de Halldórsson levou à identificação de diversos problemas e à conclusão que a mesma não é viável na prática. Apresentamos duas hipóteses que acreditamos refletem os motivos que dificultam sua utilização em redes reais.

O restante deste trabalho está organizado da seguinte maneira. A Seção 2 descreve o modelo SINR e o reuso espacial. Na Seção 3 é apresentada uma estratégia de escalonamento construída sob o modelo SINR para redes densas e na Seção 4 são discutidos os motivos que nos fazem concluir que a mesma não é praticável. Por fim, as conclusões são apresentadas na Seção 5.

\section{O Modelo SINR e o Reuso Espacial}

O modelo SINR (Signal-to-Interference-plus-Noise Ratio) também conhecido como modelo de interferência física ou modelo físico, é um modelo que considera os efeitos da interferência cumulativa na recepção do sinal, além de considerar os efeitos do desvanecimento sobre a potência do sinal transmitido. Trabalhos diversos mostram as vantagens do modelo para representar redes sem fio reais [Son et al. 2006, Maheshwari et al. 2008].

O modelo SINR estabelece um critério para que uma transmissão seja enviada, recebida e corretamente decodificada pelo receptor. Este critério é construído com base em três propriedades observadas na comunicação sem fio: a propagação do sinal (efeito de desvanecimento), a presença de ruído e a interferência entre sinais. O modelo adotado para propagação do sinal considera que a potência do sinal transmitido decai proporcionalmente ao inverso da distância entre o transmissor $i$ e o receptor $j$, representado por $d(i, j)$, elevado a um expoente denominado de path loss (perda de percurso), $\alpha$. Ruídos são sinais elétricos indesejados, os quais não são passíveis de controle e que podem interferir com os sinais desejados (sinais transmitidos com mensagens). No modelo SINR o ruído é representado por uma constante $N_{0}$. A interferência ocorre quando existem transmissões simultâneas. O modelo SINR computa o nível de interferência recebido pelo dispositivo $j$, por meio do somatório das $\tau$ transmissões simultâneas, com exceção da transmissão desejada entre $i$ e $j$.

\footnotetext{
${ }^{1}$ https://omnetpp.org/

${ }^{2}$ https://inet.omnetpp.org/
} 
O critério para considerar que uma transmissão é bem sucedida é o seguinte. A potência do sinal recebido menos a soma das potências dos sinais interferentes e a potência do ruído deve ser maior ou igual ao limiar $\gamma$, chamado de SINR threshold ou limite SINR e expresso em decibéis (dB), especificado na inequação 1.

$$
\frac{\frac{P_{T i}}{d(i, j)^{\alpha}}}{N_{0}+\sum_{\substack{k=1 \\ k \neq i}}^{\tau} \frac{P_{T k}}{d(k j)^{\alpha}}} \geq \gamma
$$

Uma importante observação deve ser feita: a premissa básica do modelo SINR indica que é a potência de interferência cumulativa que determina a possibilidade de correta recepção do sinal [Blough et al. 2010]. Assim, transmissões simultâneas entre dispositivos que compartilham um mesmo espectro de frequências podem ocorrer, com os sinais sendo recebidos corretamente, desde que o nível de interferência acumulativo não eleve a SINR a um ponto que impossibilite a recepção. Para que transmissões simultâneas ocorram, é necessário a utilização de um mecanismo ou algoritmo de escalonamento que estabeleça uma ordem em relação as transmissões.

Um algoritmo de escalonamento pode fazer uso das distâncias que separam os dispositivos para escalonar as transmissões em intervalos (slots) de tempo. O modelo permite o chamado reuso espacial, com múltiplas transmissões escalonadas para um mesmo slot. Considere, como exemplo, um par de dispositivos $i$ e $j$ que estão próximos um ao outro em comparação aos demais dispositivos da rede em que se encontram. Considere ainda que, a potência irradiada pelo dispositivo transmissor $i$ e recebida por $j$ é muito superior em relação a potência cumulativa recebida de outras transmissões simultâneas, que são consideradas, portanto, interferência. Neste exemplo $j$ pode receber o sinal de $i$ corretamente, isto é, a transmissão entre $i$ e $j$ pode ocorrer de modo concomitante a outras transmissões. Quanto maior o número de transmissões possíveis em um mesmo instante de tempo, maior é o reuso espacial. Por este motivo, o reuso espacial é inclusive utilizado como métrica de avaliação de algoritmos de escalonamento [Gore et al. 2007]. A seção a seguir descreve uma estratégia de escalonamento em redes sem fio, sob o modelo SINR, que utiliza o reuso espacial.

\section{Uma Estratégia de Comunicação para Redes Sem Fio Densas}

Esta seção descreve uma estratégia de escalonamento de transmissões em redes sem fio densas no modelo SINR proposta por Halldórsson e outros [Halldórsson et al. 2017]. A estratégia baseia-se no mecanismo de acesso TDMA (Time Division Multiple Access). Redes sem fio densas possuem uma grande quantidade de dispositivos concentrados em pequenas áreas. A alta densidade de dispositivos faz com que os protocolos e mecanismos de acesso ao meio baseados em contenção tornem-se ineficientes. Estratégias em que os dispositivos competem pelo uso exclusivo do canal de comunicação são inviáveis em redes densas, pois o tempo de espera para uso do meio inevitavelmente aumenta, causando uma diminuição da taxa de transmissão total, impactando aplicações e usuários.

A estratégia de escalonamento de Halldórsson é descrita seguir. O modelo de sistema é apresentado na Subseção 3.1. A Subseção 3.2 apresenta o conceito de grafo induzido SINR, fundamental para a obtenção da estrutura de comunicação em árvore 
apresentada na Subseção 3.3 e para o desenvolvimento da estratégia de escalonamento apresentada na Subseção 3.4 .

\subsection{Modelo de Sistema}

A estratégia de escalonamento assume uma rede densa do tipo 1-hop (single hop) na qual se um dispositivo é o único que transmite uma mensagem em um determinado intervalo de tempo, todos os demais dispositivos recebem a mensagem. A rede é representada portanto por um grafo completo $G=(V, E)$, sendo $V$ o conjunto de $n>1$ dispositivos identificados unicamente que compõem a rede e $E$ o conjunto de arestas entre todo par de dispositivos.

Assume-se também que a rede é síncrona: o tempo avança em intervalos de tempo chamados de rodadas síncronas que são rotuladas de modo incremental $(1,2,3 \ldots)$, com todos os dispositivos iniciando pela rodada 1. Considera-se que os dispositivos estão conectados por meio de uma rede sem fio que os possibilita transmitir mensagens em broadcast. Seja $d(i, j)$ a distância entre os dispositivos $i$ e $j$, assume-se que a distância mínima entre dois dispositivos quaisquer é $d_{\text {min }}$. Existem constantes $\lambda \mathrm{e} d, \lambda>0 \mathrm{e}$ $0<d<\alpha$, sendo $\alpha$ o coeficiente de path loss. Se $U$ é o conjunto composto pelos dispositivos com distância mútua mínima $d_{m i n}$, e todos os dispositivos em $U$ estão a uma distância máxima $x \cdot d_{\text {min }}$ de um dispositivo qualquer de $U$, então $|U| \leq \lambda \cdot x^{d}$. No trabalho assume-se $d=2$ e denota-se por $R$ a razão entre a maior e a menor distância entre os dispositivos contidos em $V$.

No modelo adotado não é permitido aos dispositivos adaptarem os níveis de potência de transmissão, sendo adotado um nível de potência uniforme $P_{T}$ (igual a 1) para todos. Assume-se que a potência do sinal transmitido decai proporcionalmente ao inverso da distância entre transmissor e receptor, elevados a um expoente denominado de path loss. A Inequação 1, que descreve a relação da SINR, é utilizada para calcular o raio de alcance, isto é, o máximo alcance de transmissão na ausência de demais transmissões. Para isto, ignora-se a interferência cumulativa presente na inequação (considera transmissão exclusiva e, portanto, não há interferência no denominador). Transformando a desigualdade em igualdade, obtém-se a distância máxima na qual o sinal transmitido é atenuado de forma que o valor resultante seja equivalente ao valor limite (threshold) da SINR $\gamma$. Substitui-se ainda a distância entre $i$ e $j(d(i, j))$ por $r_{s}$, sendo este a representação do raio de transmissão que é o alcance máximo de transmissão. O ruído de fundo $N_{0}$ mantém-se como na inequação original. O resultado é mostrado na Equação 2.

$$
\frac{\frac{P_{T i}}{d(i, j)^{\alpha}}}{N_{0}+\sum_{\substack{k=1 \\ k \neq i}}^{\tau} \frac{P_{T k}}{d(k, j)^{\alpha}}} \geq \gamma \quad \therefore \quad \frac{\frac{P_{T}}{r_{s}^{\alpha}}}{N_{0}}=\gamma \quad \therefore \quad r_{s}=\left(\gamma \cdot N_{0}\right)^{-1 / \alpha}
$$

\subsection{Grafo Induzido SINR}

O grafo $G$ que representa o sistema é completo porque, se não houver outra comunicação em curso, qualquer par de dispositivos pode se comunicar com sucesso. Entretanto, as comunicações interferem entre si e nem toda comunicação pode ocorrer ao mesmo tempo. O conceito de grafo induzido SINR (SINR induced graph) foi introduzido 
[Daum et al. 2013] como um passo para determinar quais pares de dispositivos podem se comunicar simultaneamente na rede. A construção do grafo induzido SINR inicia pela definição do grafo $H_{p}^{\mu}[U]$, induzido pelo conjunto de dispositivos $U$, uma probabilidade de transmissão $p$ e um parâmetro de confiabilidade $\mu \in(0, p)$. Assume-se que para um dado conjunto de dispositivos $U$, cada dispositivo neste conjunto transmite de maneira independente com probabilidade $p$. Assume-se também que não existe interferência além daquela causada pelos dispositivos em $U . H_{p}^{\mu}[U]$ é um grafo não direcionado com o conjunto de dispositivos $U$ e arestas $E_{p}^{\mu}[U]$, tal que para qualquer $i, j \in U$, a aresta $\{i, j\}$ existe em $E_{p}^{\mu}[U]$ se e somente se:

- $j$ recebe uma mensagem de $i$ com probabilidade de pelo menos $\mu$;

- $i$ recebe uma mensagem de $j$ com probabilidade de pelo menos $\mu$;

Dada a dificuldade de obter o grafo $H_{p}^{\mu}[U]$ de maneira exata, uma aproximação $\tilde{H}_{p}^{\mu}[U]=\left(U, \tilde{E}_{p}^{\mu}[U]\right)$ para $H_{p}^{\mu}[U]$ foi proposta [Daum et al. 2013]. A aproximação $\tilde{H}_{p}^{\mu}[U]$ é um grafo não direcionado e possui uma aproximação com precisão $\varepsilon$ de $H_{p}^{\mu}[U]$ se e somente se:

$$
E\left[H_{p}^{\mu}[U]\right] \subseteq E\left[\tilde{H}_{p}^{\mu}[U]\right] \subseteq E\left[H_{p}^{(1-\varepsilon) \mu}[U]\right]
$$

Os passos descritos em [Daum et al. 2013] permitem que uma aproximação com precisão $\varepsilon$ possa ser computada em $2 \Gamma$ rodadas, no qual $\Gamma=c\left(\frac{\log n}{\varepsilon^{2} \mu}\right)$ para uma constante suficientemente grande $c>0$. Estas $2 \Gamma$ rodadas são divididas em duas etapas distintas cada uma com duração $\Gamma$. Na primeira etapa todos os dispositivos em $U$ transmitem com probabilidade $p$ mensagens de descoberta (beacon). Este tipo de mensagem contém apenas o identificador (id) do dispositivo transmissor e serve para anunciar a sua presença aos demais. Ao final desta etapa todos os dispositivos compilam uma lista de potenciais vizinhos contendo os identificadores dos dispositivos dos quais receberam pelo menos $\left(1-\frac{\varepsilon}{2}\right) \mu \Gamma$ mensagens de descoberta durante as $\Gamma$ primeiras rodadas.

Na segunda etapa, os dispositivos em $U$ transmitem com probabilidade $p$ mensagens contendo a sua própria lista de potenciais vizinhos. Ao final desta etapa cada dispositivo constrói seu grafo $\tilde{H}_{p}^{\mu}[U]$. Para que uma nova aresta $\{i, j\}$ seja adicionada em $\tilde{E}_{p}^{\mu}[U]$, o dispositivo $i$ precisa possuir o dispositivo $j$ em sua lista de prováveis vizinhos e ter recebido uma mensagem de $j$ informando também tê-lo em sua lista de prováveis vizinhos. Os autores de [Daum et al. 2013] mostram que este algoritmo é capaz de computar uma aproximação com precisão $\varepsilon$ de $H_{p}^{\mu}[U]$ com uma alta probabilidade. Em [Halldórsson et al. 2017] é mostrado que os vértices conectados por arestas no grafo $H_{p}^{\mu}[U]$ estão a distâncias relativamente curtas. Para chegar a esta afirmação, os autores provam o Lema 1 a seguir.

Lema 1 Dada uma constante $\zeta \geq 1$, existe uma constante $\nu_{\zeta}>0$ de modo que a seguinte afirmação é válida. Se $U \subseteq V$ é o subconjunto composto pelos vértices (dispositivos) com distância mútua mínima $d_{\text {min }}$ e $i, j \in U$ são vértices que possuem distância $d(i, j) \leq$ $\zeta \cdot d_{\text {min }}$, então sempre que i transmitir enquanto os demais vértices com distância $\nu_{\zeta} \cdot d_{\text {min }}$ permanecerem em silêncio, é garantido que j recebe corretamente a transmissão de $i$.

Resumidamente, o Lema 1 afirma que para um par emissor-receptor, separados por uma distância máxima $d_{\zeta}=\zeta \cdot d_{\text {min }}$, é garantida a correta recepção do sinal se os demais dispositivos, que encontram-se a uma distância $d_{\nu}=\nu_{\zeta} \cdot d_{\min }$ em relação 
ao receptor permanecerem em silêncio. A prova deste lema encontra-se no apêndice de [Halldórsson et al. 2017].

A seguir, é mostrado como obter a distância $d_{\nu}$ por meio de um exemplo. Seja $U$ o conjunto composto pelos vértices $\{i, j, l, m, n, o\}$, no qual cada par de vértices está separado por uma distância mínima $d_{\text {min }}=5 \mathrm{~m}$. Os dispositivos em $U$ podem transmitir sinais com potência uniforme $P_{T}=0.625 \mathrm{~mW}$. Assume-se que o sinal desvanece com expoente de path loss $\alpha=4$ e a existência de ruído de fundo $N_{0}=-90 \mathrm{dBm}$ em cada receptor. Um sinal é recebido corretamente apenas se a SINR resultante no receptor for maior ou igual ao limite SINR $\gamma=20 \mathrm{~dB}$. Um sinal transmitido exclusivamente, ou seja, sem outras transmissões simultâneas, sob estas condições é recebido corretamente por um transmissor que encontra-se a distância máxima de $50 \mathrm{~m}$. O exemplo é ilustrado pela Figura 1 e descrita a seguir.

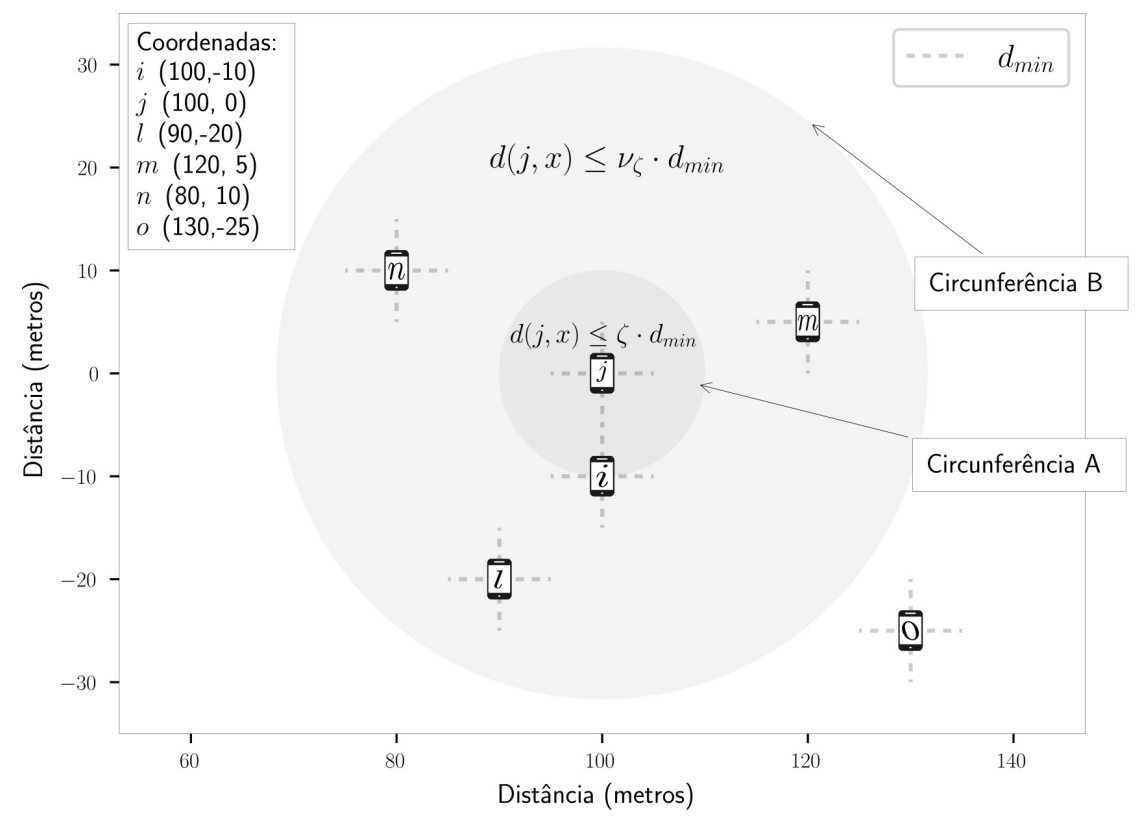

Figura 1. Exemplo para o Lema 1.

Ao centro da Figura 1 encontra-se o dispositivo $j$ envolto por duas circunferências, A e B. A circunferência A possui raio igual a $d_{\zeta}=\zeta \cdot d_{\text {min }}$. Por exemplo, para $\zeta=2$, o raio da circunferência com origem em $j$ é de $10 \mathrm{~m}$. Qualquer dispositivo $x \in U$ dentro desta circunferência está a uma distância $d(j, x) \leq \zeta \cdot d_{\text {min }}$ de $j$. Note que no limite desta circunferência existe apenas um único dispositivo, identificado como $i$. O Lema 1 afirma que uma transmissão proveniente de um dispositivo dentro da circunferência A tem sucesso se nenhum outro dispositivo dentro da circunferência B transmite simultaneamente. No caso, uma transmissão do dispositivo $i$ é recebida corretamente pelo dispositivo $j$ se os dispositivos $l, m$ e $n$ não fizerem transmissão simultânea. A circunferência B, portanto, delimita a distância $d(j, x)$ entre um dispositivo $x \in U$ e $j$, sendo $d(j, x) \leq \nu_{\zeta} \cdot d_{\text {min }}$.

$O$ raio da circunferência $B$ pode ser obtido por meio da inequação do modelo SINR, como mostrado a seguir. Considere uma situação na qual duas transmissões ocorrem simultaneamente. A primeira transmissão consiste no sinal considerado desejado, tendo como origem o dispositivo $i$ e destino o dispositivo $j$. Assim como na Figura 1, assume-se que $i$ está a uma distância $d_{\zeta}=\zeta \cdot d_{\min }$ de $j$, isto é, $i$ está no limite da circun- 
ferência A. A segunda transmissão é feita pelo dispositivo $x \in U$ e tida como interferência para o sinal desejado. Neste sentido, busca-se descobrir qual é a distância mínima $d_{\nu}$ que $x$ deve manter de $j$ para que a SINR resultante do sinal de $i$ seja superior ao limite SINR em $j$. Esta distância é obtida por meio da inequação da SINR 1. O cálculo é mostrado abaixo pela Equação 4. Na segunda inequação, a distância entre $i$ e $j$ é substituída por $d_{\zeta}$ no numerador da fração. Ao invés de considerar um somatório de transmissões interferentes, assume-se uma única transmissão interferente no denominador da fração, com distância $d_{\nu}$ em relação ao receptor do sinal. Por fim, a última inequação é escrita em função de $d_{\nu}$, i.e, esta inequação estabelece o raio da circunferência B. Portanto, desta maneira, é possível obter a distância mínima $d_{\nu}$ em relação a $j$ na qual os dispositivos, com exceção de $i$, devem permanecer em silêncio para que comunicação entre $i$ e $j$ tenha sucesso, sendo $d_{\nu}=d(j, x) \leq \nu_{\zeta} \cdot d_{\text {min }}$.

$$
\frac{\frac{P_{T i}}{d(i, j)^{\alpha}}}{N_{0}+\sum_{\substack{k=1 \\ k \neq i}}^{\tau} \frac{P_{T k}}{d(k, j)^{\alpha}}} \geq \gamma \quad \therefore \quad \frac{\frac{P_{T}}{d_{\zeta}^{\alpha}}}{N_{0}+\frac{P_{T}}{d_{\nu}^{\alpha}}} \geq \gamma \quad \therefore \quad d_{\nu} \geq \sqrt[\alpha]{\frac{\gamma \cdot P_{T}}{\frac{P_{T}}{d_{\zeta}^{\alpha}}-\gamma \cdot N_{0}}}
$$

Os autores de [Halldórsson et al. 2017] estendem o Lema 1 para demonstrar que $H_{p}^{\mu}[U]$ contém todas as arestas que representam pares de vértices com distâncias relativamente curtas. Este novo lema de [Halldórsson et al. 2017] é o Lema 2 a seguir.

Lema 2 Para todo $p \in(0,1 / 2]$ e toda constante $\eta>1$, existe $\mu \in(0, p)$ tal que $\forall U \subseteq$ $V$ com distância mínima $d_{\text {min }}$ entre os pares, o grafo $H_{p}^{\mu}[U]$ (e assim também o grafo $\left.\tilde{H}_{p}^{\mu}[U]\right)$ contém todas arestas entre os vértices $i, j \in U \operatorname{com} d(i, j) \leq \eta \cdot d_{\text {min }}$.

Em linhas gerais, este lema é demonstrado da seguinte maneira. Seja $\nu$ uma constante para qual o Lema 1 é verdadeiro para $\zeta=\eta$. Seja $i, j \in U$ um par de vértices quaisquer com $d(i, j) \leq \eta \cdot d_{\text {min }}$. Seja $S$ o conjunto de vértices em $V$ com distância $\nu \cdot d_{\text {min }}$ em relação a $j$, excluindo $i$. De acordo com as premissas associadas ao modelo de sistema, $|S| \leq \lambda \cdot \nu^{d}$. Pelo Lema $1, j$ recebe uma mensagem de $i$ se $i$ transmite enquanto os demais vértices em $S$ permanecem em silêncio. A probabilidade do primeiro evento acontecer é igual a $p$ enquanto que a probabilidade do segundo ocorrer é $(1-p)^{|S|}$. Uma vez que estes dois eventos são independentes, segue que $j$ recebe corretamente a mensagem de $i$ com probabilidade $p(1-p)^{|S|} \geq p(1-p)^{\lambda \cdot \nu^{d}}$. Pelo principio da simetria, o mesmo é válido para que $i$ receba corretamente uma mensagem de $j$. Consequentemente, para $\mu \geq p^{2}(1-p)^{2 \lambda \cdot \nu^{d}},\{i, j\}$ satisfaz a condição de ser uma aresta em $H_{p}^{\mu}$.

\section{3. Árvore de Torneio}

A estratégia de escalonamento ainda utiliza a chamada árvore de "torneio". A árvore é chamada de "torneio" justamente porque um nodo não-folha representaria o "vencedor" de uma competição entre seus filhos. Uma árvore de torneio $T$ definida sobre um conjunto de dispositivos $V$ deve satisfazer as seguintes propriedades:

1. Todos os nodos folha estão no mesmo nível na árvore.

2. Cada nodo da árvore corresponde a um dispositivo de $V$.

3. Os $n$ nodos folha correspondem univocamente aos $n$ dispositivos de $V$. 
4. Cada nodo não-folha corresponde a um dispositivo representado por um de seus filhos.

A Figura 2 exibe uma representação de uma árvore de torneio $T$. Os níveis $\ell$ da árvore $T$ são enumerados a partir da raiz, que recebe o valor zero, aumentando o valor atribuído ao nível conforme move-se para os nodos folha. Define-se $h(T)$ para descrever a altura da árvore $T$. Para um nível $\ell \in[0, h(T)], V(T, \ell)$ é o conjunto de dispositivos que correspondem ao nodos daquele nível $\ell$.

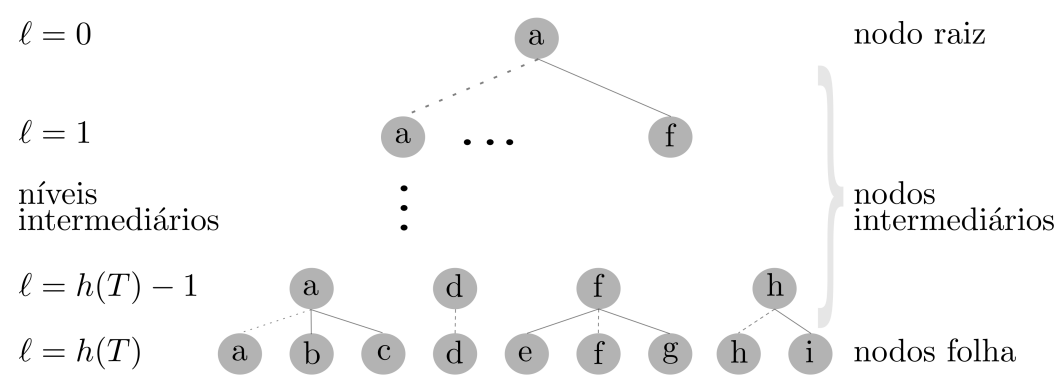

Figura 2. Exemplo de uma árvore de torneio $T$.

Os dispositivos podem se comunicar depois que a árvore é construída. A comunicação é organizada em fases, cada fase dedica-se a um único nível da árvore e é composta por múltiplas rodadas. Um nodo pode se comunicar com seus pais ou filhos na árvore apenas na fase dedicada a seu nível e em sua respectiva rodada. Tendo terminado uma fase, inicia-se a próxima fase dedicada ao nível $\ell$ imediatamente superior em $T$, isto acontece até que se alcance o nível $\ell=0$. Após a fase dedicada ao nível do nodo raiz, inicia-se um novo ciclo tendo como início novamente o nível mais baixo $\ell=h(T)$ da árvore $T$.

Para a construção da árvore de torneio $T$ é necessário definir a probabilidade de transmissão $p$ e o parâmetro de confiabilidade $\mu$, tal que as condições para o Lema 2 sejam satisfeitas para $\eta=2$ e o grafo induzido $H_{p}^{\mu}[U]$ contenha todas as arestas de distância máxima $2 \cdot d_{\min }$ para todo $U \subseteq V$. A construção da árvore é feita um nível $\ell$ por vez, iniciando-se pelo nível mais baixo $\ell=h(T)$ até que se alcance nível $\ell=1$. O cômputo de cada nível produz como saída o conjunto de dispositivos $V(T, \ell-1)$, utilizado para construção do nível $\ell-1$ (nível imediatamente superior na árvore). Os passos para a construção de cada nível $\ell \in\{1, \ldots, h(T)\}$ são:

1. Computa-se o grafo $H_{\ell}:=\tilde{H}_{p}^{\mu}[V(T, \ell)]$ da forma descrita na Seção 3.2.

2. Computa-se o conjunto independente maximal (Maximal Independent Set - MIS) $I$ de $H_{\ell}$. Os nodos de $V(T, \ell-1)$ são exatamente os nodos de $I$.

3. Cada nodo $i \in V(T, \ell) \backslash V(T, \ell-1)$ escolhe seu nodo pai de modo arbitrário entre aqueles que são seus vizinhos em $H_{\ell}$ e estão em $V(T, \ell-1)$.

O Teorema 1 apresenta propriedades de uma árvore de torneio $T$, construída utilizando os passos descritos acima [Halldórsson et al. 2017].

Teorema 1 Com alta probabilidade, T satisfaz as seguintes propriedades:

- A árvore T pode ser construída em tempo poli-logarítmico no modelo SINR. 
- A altura da árvore é $h(T)=\mathcal{O}(\log R)$, no qual $R$ é a razão entre a maior e a menor distância entre os dispositivos de $V$.

- Suponha que todos os dispositivos em $V(T, \ell)$ de um determinado nível $\ell \in h(T)$ transmitem em uma rodada $r$ com probabilidade p e os outros dispositivos dos outros níveis permanecem em silêncio. Então para todo dispositivo $i \in V(T, \ell), i$ se comunica com sucesso com seu pai na rodada $r$ com probabilidade constante.

Resumidamente, a prova do Teorema 1 é feita da maneira seguinte. Seja $d_{\min }^{(\ell)}$ a distância mínima entre os vértices em $V(T, \ell)$. Por meio do Lema 2 e da escolha dos parâmetros $p$ e $\mu$, o grafo $H_{\ell}$ contém uma aresta para cada par de vértices $i, j \in V(T, \ell)$ que estão a distância máxima $\min \left\{2 \cdot d_{\text {min }}^{(\ell)}, r_{s}\right\}$, sendo $r_{s}$ o alcance de transmissão. Uma vez que os vértices em $V(T, \ell-1)$ são os vértices obtidos a partir do cômputo do conjunto independente maximal do grafo $H_{\ell}$, não existem dois vértices em $V(T, \ell-1)$ que possam ser vizinhos em $H_{\ell}$, deste modo, tem-se que $d_{\min }^{(\ell-1)}>\min \left\{2 \cdot d_{\min }^{(\ell)}, r_{s}\right\}$. Portanto, desde que $d_{\text {min }}^{(l)} \leq r_{s} / 2$, tem-se que $d_{\text {min }}^{(\ell-1)}>2 \cdot d_{\text {min }}^{(\ell)}$. Como $d_{m i n}^{(\ell)}>r_{s} / 2$, pelo Lema $2, H_{\ell}$ é um grafo completo e deste modo é possível alcançar o nível $\ell=1$. A afirmação de que $h(T)=\mathcal{O}(\log R)$ se confirma porque a partir da definição de $R$, a distância mínima entre os vértices em $V$ é de ao menos $r_{s} / R$. A terceira propriedade se refere à situação em que os dispositivos do nível 1 transmitem com probabilidade $p$ enquanto os demais ficam em silêncio. A propriedade diz que tanto um dispositivo deste nível recebe mensagem do pai ou seu pai recebe mensagem do dispositivo com probabilidade constante. Lembre-se que os pais dos dispositivos do nível $l$ também estão no nível $l$, pelas regras de construção da árvore de torneio.

\subsection{Escalonamento TDMA Baseado em Árvore de Torneio}

A estratégia de escalonamento TDMA é construída sobre a árvore de torneio $T$. Inicialmente, definem-se os parâmetros $p, \mu$ e $\varepsilon$. Cada nível $\ell$ da árvore de torneio é então construído, um nível por vez, começando pelo nível mais baixo $\ell=h(T)$, até o nível $\ell=1$. Para definir cada nível da árvore é construído o grafo induzido $H_{\ell}=\tilde{H}_{p}^{\mu}[V(T, \ell)]$. No grafo $H_{\ell}$ é possível a comunicação entre os vértices adjacentes. $\mathrm{O}$ escalonamento $S_{\ell}$ construído para cada nível $\ell$ é composto por $L$ rodadas. Um algoritmo de coloração é usado para definir o escalonamento dos vértices de $H_{\ell}$. A coloração corresponde a um problema de rotulagem, no qual é atribuído um rótulo, tradicionalmente uma cor, a um elemento (vértice ou aresta) sujeito a algum tipo de restrição [Matula et al. 1972]. A coloração busca colorir os vértices de tal modo que não haja dois vértices adjacentes que compartilhem a mesma cor.

Para o escalonamento, [Halldórsson et al. 2017] utilizam o algoritmo de coloração $k$-colorível (ou $k$-coloring), este algoritmo utiliza no máximo $k$ cores, sendo $k$ igual a $\Delta\left(H_{\ell}+1\right)$, no qual $\Delta$ é o grau máximo do grafo. Em cada nível $\ell$ é definido o conjunto de cores $\phi_{\ell}$. Isto quer dizer que para um determinado nível $\ell$ é definido um escalonamento $S_{\ell}$ no qual cada vértice $i \in V(T, \ell)$ recebe uma cor $\phi_{\ell}(i) \in\{1, \ldots, L\}$. Somente os dispositivos $i \in V(T, \ell)$ com $\phi_{\ell}(i)=r$, transmitem na rodada $r$. Uma aresta $\{i, j\} \in$ $E\left[H_{\ell}\right]$ é dita bem sucedida no que diz respeito ao escalonamento $S_{\ell}$ se na rodada $\phi_{\ell}(i)$ do escalonamento $S_{\ell}, j$ recebe uma mensagem de $i$, e na rodada $\phi_{\ell}(j)$ de $S_{\ell}, i$ recebe uma mensagem de $j$. A partir de então, como próximo passo, define-se $H_{\ell}^{\prime}$ como o subgrafo de $H_{\ell}$ composto por todas as arestas consideradas bem sucedidas em relação a $S_{\ell}$. O grafo $H_{\ell}^{\prime}$, e não mais o grafo $H_{\ell}$, é utilizado para a construção do escalonamento. 
De maneira resumida, os passos para a construção de um nível $\ell \in\{1, \ldots, h(T)\}$ são:

1. Computa-se o grafo $H_{\ell}:=\tilde{H}_{p}^{\mu}[V(T, \ell)]$, como descrito na Seção 3.2.

2. Aplica-se o algoritmo de coloração sobre o grafo $H_{\ell}$.

3. Computa-se o grafo $H_{\ell}^{\prime}$, subgrafo de $H_{\ell}$, com $E\left[H_{\ell}\right]$ consistindo em todas as arestas de $H_{\ell}$ que são bem sucedidas no que diz respeito ao escalonamento $S_{\ell}$.

4. Computa-se o conjunto independente maximal $I$ de $H_{\ell}^{\prime}$. Os nodos de $V(T, \ell-1)$ são exatamente os nodos de $I$.

5. Cada nodo $i \in V(T, \ell) \backslash V(T, \ell-1)$ escolhe seu nodo pai de modo arbitrário entre aqueles que são seus vizinhos em $H_{\ell}^{\prime}$ e estão em $V(T, \ell-1)$.

Estes passos permitem, portanto, a obtenção de uma estratégia de comunicação para redes densas que utiliza-se do reuso espacial, uma vez que múltiplas transmissões simultâneas são escalonadas para a mesma rodada. A árvore define fases, cada fase é dedicada a um único nível $\ell$ de $T$. As fases são executadas no sentido folhas para raiz. Cada uma destas fases divide-se em $L$ rodadas, sendo $L$ o número total de cores utilizadas no nível. Em uma fase, as rodadas $r \in\{1, \ldots, L\}$ são executadas de maneira sequencial e somente os dispositivos coloridos com a respectiva cor da atual rodada podem transmitir mensagens.

\section{Argumentos sobre a Inviabilidade da Estratégia de Escalonamento}

Para o desenvolvimento do presente trabalho foi implementado um simulador de redes sem fio sob o modelo SINR. A implementação fez uso do simulador OMNET++ e do framework INET. A implementação da estratégia de escalonamento descrita acima por meio de simulação nos trouxe indícios de sua inviabilidade na prática. Apenas em cenários muito raros a estratégia se mostrou efetiva. A partir da observação dos resultados, temos duas hipóteses para a afirmar que a estratégia de escalonamento não é aplicável na prática, as duas subseções a seguir discutem as hipóteses:

1. A dificuldade da escolha dos parâmetros de probabilidade de transmissão $p$ e de confiabilidade $\mu$ para que a aproximação do grafo induzido SINR $\tilde{H}_{p}^{\mu}[U]$, contenha todas as arestas de distância máxima $2 \cdot d_{\text {min }}$.

2. O algoritmo de coloração $k$-colorível não é capaz de escalonar adequadamente as transmissões do grafo $H_{\ell}=\tilde{H}_{p}^{\mu}[V(T, \ell)]$ para uma rede densa.

\subsection{Parâmetros para a Construção do Grafo Induzido SINR}

Uma das etapas para o escalonamento consiste em construir o grafo induzido SINR $H_{\ell}=H_{p}^{\mu}[V(T, \ell)]$, induzido pelo conjunto de dispositivos $U$, a probabilidade de transmissão $p$ e o parâmetro de confiabilidade $\mu$. Na verdade uma aproximação é utilizada, o grafo $\tilde{H}_{p}^{\mu}[V(T, \ell)]$ descrito na Seção 3.2. O algoritmo de construção do grafo consiste em duas etapas com duração de $\Gamma$ rodadas cada. Na primeira etapa, todos transmitem com probabilidade $p$ mensagens de descoberta indicando a sua presença. Na segunda etapa, os dispositivos anunciam quais são seus prováveis vizinhos. O critério utilizado para ser um provável vizinho é ter recebido ao menos $\Gamma \mu$ mensagens de descoberta. Para que uma aresta $\{i, j\}$ seja adicionada em $\tilde{E}_{p}^{\mu}[U]$, o dispositivo $i$ precisa considerar $j$ seu provável vizinho e ter recebido ao menos uma mensagem de $j$ informando que $i$ é seu provável vizinho também. 
Os autores de [Halldórsson et al. 2017] afirmam que este algoritmo constrói um grafo $\tilde{H}_{p}^{\mu}[V(T, \ell)]$ que contém todas as arestas entre os dispositivos que estão a distância máxima $2 \cdot d_{\text {min }}$. Para satisfazer a esta propriedade é necessário determinar valores adequados para os parâmetros $p$ (probabilidade de transmissão) e $\mu$ (confiabilidade) que atendem a esta condição. Não há método analítico para determinar os valores, descrevemos a seguir como tirar proveito da simulação para determinar os parâmetros.

Foi estabelecido como critério para a correta recepção do sinal o limite SINR $\gamma=20 \mathrm{~dB}$. Foram simulados três sistemas, com 10, 20 e 30 dispositivos. As distâncias máximas entre pares de dispositivos foram de 50, 100 e 150 metros, respectivamente. A distribuição destes dispositivos no plano euclidiano ocorreu de maneira aleatória, respeitando uma distância mínima de 5 metros entre cada par de dispositivo. Para cada um destes sistemas, foram simulados 300 cenários distintos, mudando o posicionamento dos dispositivos. A potência de transmissão foi ajustada de modo que toda transmissão exclusiva tem sucesso.

Inicialmente, foi realizada uma avaliação do impacto do parâmetro $p$ sobre a quantidade de mensagens recebidas. São utilizados dois rótulos para mensagens recebidas: short edge e long edge. O rótulo short edge é atribuído para as mensagens recebidas de dispositivos que encontram-se a uma distância menor ou igual a $2 \cdot d_{\min }$ do receptor, relembrando que $d_{\min }$ é a menor distância entre um par qualquer de dispositivos do cenário. Todas as demais mensagens recebem o rótulo de long edge. O resultado é apresentado na Figura 3. Os gráficos no formato de barras, mostram:

- $\min \{$ short edge\}: A quantidade mínima de mensagens short edge recebidas em cada uma das simulações.

- media\{short edge\}: A quantidade média de mensagens short edge recebidas.

- media\{long edge\}: A quantidade média de mensagens long edge recebidas.
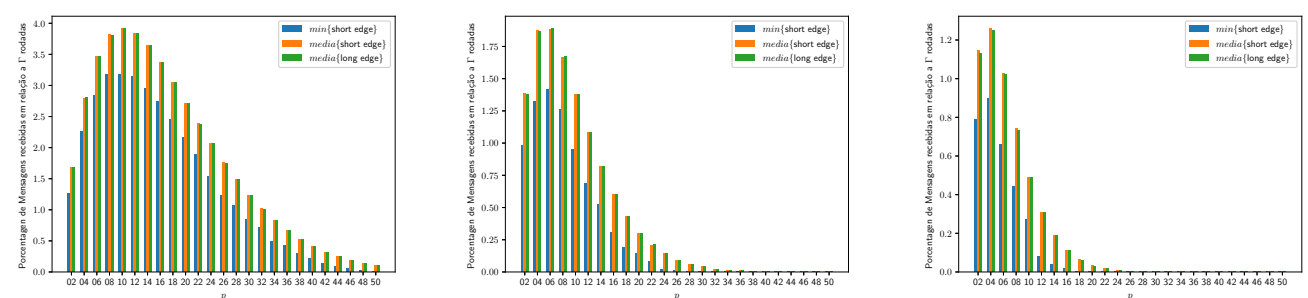

Figura 3. Avaliação do parâmetro $p$ em sistemas com 10, 20 e 30 dispositivos.

No eixo das abscissas está a probabilidade de transmissão $p$. O eixo das ordenadas indica a porcentagem de mensagens recebidas em relação a quantidade de $\Gamma$ rodadas, nas quais os dispositivos tiverem a possibilidade de comunicar. Inicialmente, examinando a Figura 3, nota-se que quanto maior a quantidade de dispositivos, maior a restrição em relação aos valores de $p$ que podem ser utilizados. Um valor de $p=28$, por exemplo, pode fazer com que uma quantidade de mensagens considerável seja recebida pelo sistema que possui 10 dispositivos. Entretanto, o mesmo valor para um sistema com 30 dispositivos, representa uma alta probabilidade de muitos dispositivos transmitirem na mesma rodada. Isto faz com que a interferência mútua seja tamanha ao ponto de impossibilitar qualquer recepção correta. Ao observar as simulações, nota-se que em um ambiente denso, no qual 
os dispositivos utilizam potências de transmissão uniformes, capazes de alcançar qualquer outro dispositivo, são pouquíssimas as situações nas quais três ou mais dispositivos transmitem em simultâneo e estas mensagens são recebidas corretamente por alguns dos demais dispositivos.

Atribuir um valor para o parâmetro $\mu$, de modo a atender o requisito da estratégia, é tão desafiador quanto atribuir um valor adequado para $p$. Como mostra a Figura 3, não existe uma disparidade notável entre as médias de mensagens do tipo short edge e long edge, ao ponto de possibilitar traçar um limiar de confiança para $\mu$, e assim conseguir identificar quais são os dispositivos que encontram-se próximos. Sendo fundamentais para a realização do escalonamento, a indefinição sobre valores adequados de $p$ e $\mu$ tornase um forte fator impeditivo.

\subsection{Impossibilidade de Coloração em Redes Densas}

A segunda hipótese consiste na afirmação de que um algoritmo de coloração $k$-colorível não é apropriado para o escalonamento de uma rede sem fio densa tal como apresentado em [Halldórsson et al. 2017]. Um dos passos da estratégia consiste em aplicar o algoritmo de coloração sobre o grafo $H_{\ell}=\tilde{H}_{p}^{\mu}[V(T, \ell)]$. Deste modo, as cores atribuídas aos dispositivos determinam as respectivas rodadas em que devem transmitir. $\mathrm{O}$ objetivo é garantir que a comunicação entre os dispositivos representados por vértices adjacentes ocorra com sucesso. Para argumentar os desafios da utilização do algoritmo de coloração neste contexto, é apresentado a seguir um exemplo. Para o exemplo, é utilizada uma aproximação $\tilde{H}_{p}^{\mu}[V(T, \ell)]$ de $H_{p}^{\mu}[V(T, \ell)]$, da mesma maneira descrita em [Halldórsson et al. 2017]. O conjunto de arestas $\tilde{E}_{p}^{\mu}[U]$ é obtido a partir de uma execução da simulação. Portanto, existe uma aresta entre $i$ e $j \in U$ apenas se estes dispositivos passam a ser vizinhos durante a execução do algoritmo.

O cenário utilizado é composto por um conjunto de dispositivos $U=$ $\{0,1,2,3,4,5,6,7,8,9\}$. Os dispositivos são distribuídos aleatoriamente sobre o plano sob a condição de manterem uma distância mínima entre si de 5 metros, e uma distância máxima entre si de $50 \mathrm{~m}$. Todos os dispositivos transmitem com potência uniforme. A rede é de 1-hop: toda transmissão feita de maneira exclusiva é recebida por qualquer outro dispositivo situado a distância máxima de $50 \mathrm{~m}$. Como critério para correta recepção do sinal, estabelece-se o limite SINR $\gamma$ de 20dB. Neste cenário em particular, a menor distância $d_{\min }$ entre os vértices é de $5.65 \mathrm{~m}$, e ocorre entre os vértices " 5 " e "7". Após a construção de $H_{\ell}$ para cada um dos exemplos, é aplicado o algoritmo de coloração $k$-colorível, utilizando o seguinte conjunto de cores $\phi=\{$ "azul", "vermelho", "verde", "amarelo" $\}$.

O algoritmo descrito na Seção 3.2, para o cômputo de uma aproximação $\tilde{H}_{p}^{\mu}[V(T, \ell)]$ do grafo induzido $H_{p}^{\mu}[V(T, \ell)]$, não é tão preciso ao ponto de $\tilde{E}_{p}^{\mu}[U]$ possuir somente arestas entre os vértices $i$ e $j$ que estão separados por uma distância $d(i, j) \leq 2 \cdot d_{\min }$. Ocorre que os dispositivos consideram como vizinhos também outros dispositivos que encontram-se em distâncias maiores. $H_{\ell}$ neste exemplo é um grafo induzido sobre o conjunto de dispositivos $U$ e parâmetros $p$ e $\mu$, resultado de uma única execução da simulação. É importante ressaltar que, devido ao fato dos dispositivos transmitirem sob uma probabilidade $p$, o grafo resultante de $H_{\ell}$ pode ser diferente em cada execução do algoritmo.

A Figura 4 mostra que o algoritmo de coloração atribuiu a cor "vermelha" para 

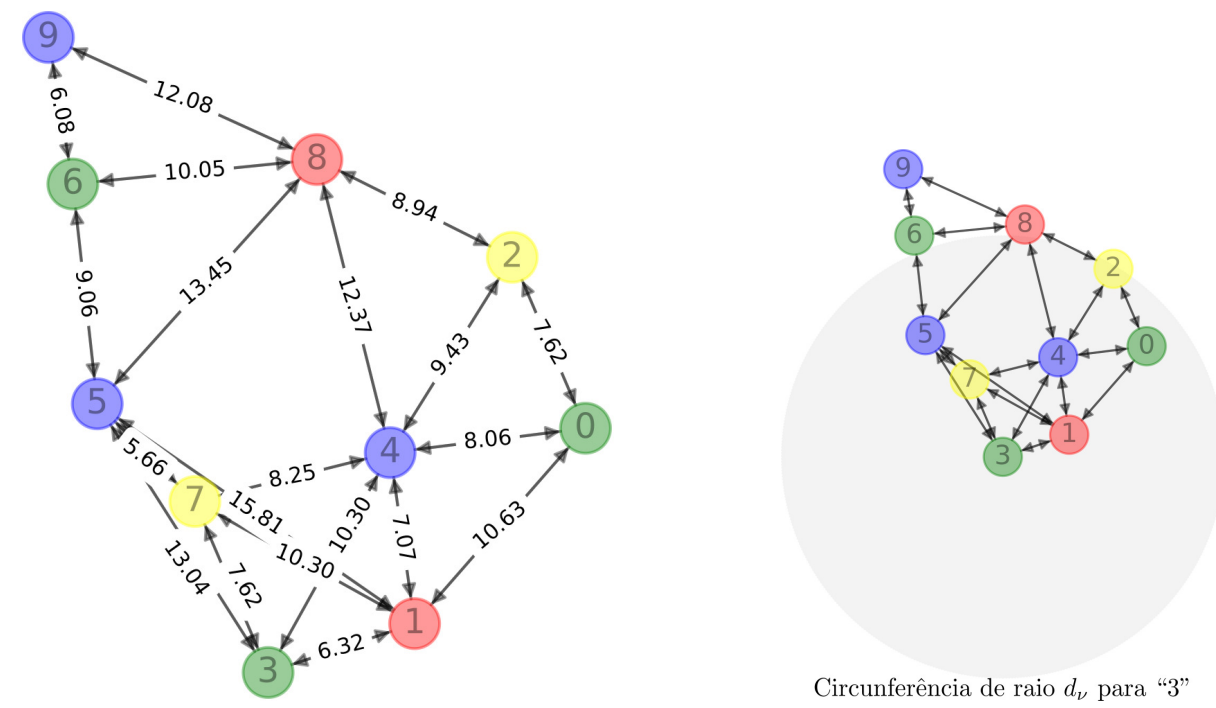

Figura 4. Esquerda: Grafo induzido $H_{\ell}$ e Direita: Comunicação entre 1 e 3.

os dispositivos "1" e "8", uma vez que estes não possuem arestas adjacentes. Para o escalonamento, isso significa que estes dispositivos (que tem a mesma cor) transmitem na mesma rodada, e os vértices adjacentes deveriam receber corretamente seu sinal. Da mesma forma, os dispositivos adjacentes a cada um deles transmitem em suas respectivas cores e suas mensagens devem ser recebidas por " 1 " e "8". Entretanto, isto não se confirma: a recepção correta não acontece como esperado. Toma-se, como exemplo, o dispositivo "1". Espera-se que "1" ao transmitir em sua respectiva cor, tenha seu sinal recebido corretamente pelos dispositivos adjacentes em $H_{\ell}$, que são os dispositivos " 0 ", "3", "4", "5" e "7". A seguir vemos o que acontece na verdade.

A parte direita da Figura 4 mostra a circunferência na qual nenhum dispositivo deve transmitir para que uma transmissão de " 1 " para " 3 " tenha sucesso. O raio da circunferência relaciona-se diretamente a distância entre o par transmissor-receptor considerado, quanto maior a distância entre eles, maior é a circunferência no qual não devem haver outras transmissões. Neste caso específico, ao escalonar em simultâneo as transmissões de " 1 " e " 8 " (ambos rotulados na cor vermelha) somente o dispositivo " 3 " recebe corretamente o sinal de " 1 ". Todas as demais transmissões fracassam.

Mesmo a aresta entre " 1 " e " 3 " não é bem sucedida. A estratégia de escalonamento define uma aresta $\{i, j\} \in E\left[H_{\ell}\right]$ como bem sucedida no que diz respeito ao escalonamento $S_{\ell}$ se na rodada $\phi_{\ell}(i)$ do escalonamento $S_{\ell}, j$ recebe uma mensagem de $i$, e na rodada $\phi_{\ell}(j)$ de $S_{\ell}, i$ recebe uma mensagem de $j$. Isto implica neste exemplo, que para que o enlace $\{1,3\}$ seja considerado bem sucedido em relação ao escalonamento, "1" também deve receber corretamente o sinal de "3", na rodada em que "3" transmite. Note, por meio da Figura 4, que o dispositivo "3" foi escalonado para transmitir em simultâneo com os dispositivos " 0 " e " 6 " (cor verde). Verifica-se, neste momento, se o sinal de " 3 " pode ser recebido por " 1 " neste escalonamento feito por coloração. Para isto, é avaliada a distância $d_{\nu}$, dos dispositivos em cor verde em relação ao dispositivo " 1 ". O dispositivo " 0 " encontra-se a uma distância inferior a $d_{\nu}$ de " 1 ” logo, as transmissões de " 3 " e " 0 " tem tamanha interferência que é possível afirmar que o dispositivo " 1 " não recebe corretamente o sinal de " 3 ". O exemplo mostrou, portanto, que dispositivo " 1 " não 
possui nenhuma aresta bem sucedida para comunicar no escalonamento em questão.

Poder-se-ia alegar que os parâmetros utilizados é que induziram a este resultado. Contra-argumentamos que quaisquer parâmetros $p$ e $\mu$ que tornam possível a construção do grafo induzido $H_{\ell}$, mesmo que tenha apenas arestas entre dispositivos que possuem distância mútua inferior ou igual a $2 \cdot d_{\text {min }}$, resultam no mesmo tipo de resultado. Há fortes indícios que o escalonamento feito por coloração, feito sobre um grafo oriundo das relações sinal ruído mais interferência, não funciona na prática. Isto porque, frequentemente são escalonados para uma mesma rodada, dispositivos que produzem interferência mútua que impossibilita que suas transmissões tenham sucesso.

\section{Conclusão}

O modelo SINR para redes sem fio tem recebido destaque, na medida em que permite o reuso espacial. No artigo argumentamos que a estratégia de escalonamento proposta por Halldorsson e outros [Halldórsson et al. 2017] não pode ser aplicada em redes reais. Utilizando simulação constatamos que é notável a dificuldade em definir valores adequados para os parâmetros de forma que a saída seja a esperada. Além disso, o uso de coloração não se mostrou capaz de garantir o reuso espacial uma rede densa. Trabalhos futuros incluem, além de uma formalização dos argumentos apresentados no artigo, a proposta de uma nova estratégia de escalonamento que sim, possa ser usada em redes reais.

\section{Referências}

Blough, D. M., Resta, G., and Santi, P. (2010). Approximation algorithms for wireless link scheduling with sinr-based interference. IEEE/ACM Trans. Netw., 18(6):1701-1712.

Daum, S., Gilbert, S., Kuhn, F., and Newport, C. (2013). Broadcast in the ad hoc sinr model. In Afek, Y., editor, Distributed Computing, pages 358-372, Berlin, Heidelberg. Springer Berlin Heidelberg.

Gore, A. D., Karandikar, A., and Jagabathula, S. (2007). On high spatial reuse link scheduling in stdma wireless ad hoc networks. In IEEE GLOBECOM 2007 - IEEE Global Telecommunications Conference, pages 736-741.

Halldórsson, M. M., Kuhn, F., Lynch, N., and Newport, C. (2017). An Efficient Communication Abstraction for Dense Wireless Networks. In 31st International Symposium on Distributed Computing (DISC 2017), pages 25:1-25:16.

Halldórsson, M. M. and Tonoyan, T. (2019). Plain sinr is enough! In Proceedings of the 2019 ACM Symposium on Principles of Distributed Computing, PODC '19, pages 127-136, New York, NY, USA. Association for Computing Machinery.

Maheshwari, R., Jain, S., and Das, S. R. (2008). A measurement study of interference modeling and scheduling in low-power wireless networks. In The 6th ACM Conference on Embedded Network Sensor Systems, SenSys'08, page 141-154.

Matula, D. W., Marble, G., and Isaacson, J. D. (1972). Graph coloring algorithms. In Graph Theory and Computing, pages 109-122. Academic Press.

Son, D., Krishnamachari, B., and Heidemann, J. (2006). Experimental study of concurrent transmission in wireless sensor networks. In Proceedings of the 4th International Conference on Embedded Networked Sensor Systems, SenSys'06, page 237-250. 